\author{
MARIA TORRES-BAGUR ${ }^{a}$ \\ ANNA RIBAS \\ JOSEP VILA-SUBIRÓSc
}

\title{
USOS DEL AGUA Y PRÁCTICAS DE AHORRO HÍDRICO DE LOS TURISTAS EN LA CUENCA DEL RÍO MUGA (GIRONA)
}

\begin{abstract}
RESUMEN
El agua es uno de los principales recursos naturales de los que depende el turismo, más aún en el Mediterráneo, dónde la escasez de agua se ve agravada por los efectos del cambio climático y el aumento creciente de la demanda.

Este artículo tiene como objetivo analizar el nivel de concienciación ambiental y el comportamiento de los turistas que se alojan en hoteles, campings y casas de turismo rural de la cuenca del río Muga (Girona, España) en relación con el uso eficiente y las prácticas de ahorro hídrico durante su estancia. Metodológicamente la investigación se basa en la realización de 580 encuestas a clientes de estos alojamientos. A partir de la información recogida se ha desarrollado un análisis clúster del cual han resultado tres perfiles diferenciados de turistas: muy concienciados y muy activos, concienciados y activos y no concienciados y poco activos. Factores como la tipología del alojamiento, su localización y las características sociodemográficas de los turistas han resultado ser factores clave para la determinación de esta clasificación tipológica.
\end{abstract}

Palabras clave: alojamientos; análisis clúster; consumo de agua; escasez de agua; Mediterráneo.

a Universidad de Girona e Instituto de Medio Ambiente, Pl. Ferrater Mora 1 17001, Girona maria.torres@udg. edu https://orcid.org/0000-0002-2579-3954.

b Universidad de Girona e Instituto de Medio Ambiente, Pl. Ferrater Mora 1 17001, Girona anna.ribas@udg.edu https://orcid.org/0000-0001-5163-0561.

c Universidad de Girona e Instituto de Medio Ambiente, Pl. Ferrater Mora 1 17001, Girona josep.vila@udg.edu https://orcid.org/0000-0001-7746-9227.

Fecha de recepción: 6/3/20. Fecha de aceptación:12/6/20. 


\title{
WATER USES AND WATER SAVING PRACTICES OF TOURISTS IN THE MUGA RIVER BASIN (GIRONA)
}

\begin{abstract}
Water is one of the most important natural resources for tourism activity. This correlation is even more important in Mediterranean basin because the decrease of water availability can be aggravated due to the climate change and the increase of its demand.

The aim of this paper is to know the behavior related to water-saving habits and practices of tourists accommodated on hotels, campsites, and rural lodgings. The methodology relies on compellation of 580 questionnaires to tourists on these accommodations. With these questionnaires, a cluster analysis has been carried out. Results suggest the differentiation of three important tourist profiles: very aware and very active; aware and active, and no aware and little active. This classification is explained due to some factors such as the type of establishment, its location or sociodemographic tourist characteristics.

KeYwords: cluster analysis; lodging industry; Mediterranean; tourists; water consumption; water scarcity.
\end{abstract}

\section{INTRODUCCIÓN}

El agua es uno de los recursos fundamentales para el desarrollo de la actividad turística (Gössling y Peeters, 2015; Morrison y Pickering, 2013; Gabarda-Mallorquí, Garcia y Ribas, 2017). Sin embargo, nos encontramos en un momento en que urge fomentar medidas destinadas a su ahorro a fin de asegurar la disponibilidad y sostenibilidad futura (Hadjikakou et al., 2015). Especialmente fruto del aumento de la demanda derivada del incremento del número de turistas, pero también de la reducción de su disponibilidad como consecuencia de los efectos del cambio climático (sequías más intensas y frecuentes, disminución de las precipitaciones, etc.) (Gössling y Peeters, 2015; Köberl, Prettenthaler y Bird, 2016) se augura un futuro a corto plazo de proliferación de las tensiones y conflictos entre sectores sociales y económicos ante la competencia creciente por este recurso (Alonso, 2008; Gössling y Hall, 2015; Gössling et al., 2012), consecuencia también de su sobreexplotación (“UNEP,” 2019). Se hace imprescindible, pues, fomentar la concienciación de todos los actores del sector turístico ante este reto ambiental de primer orden a fin de implantar buenas prácticas en el consumo y el uso eficiente de agua (Bohdanowicz, 2006; Tekken y Kropp, 2015).

Este artículo se divide en 9 apartados, incluyendo esta breve introducción. En primer lugar, se presenta el marco teórico determinando los estudios que han realizado investigaciones similares a la que aquí se presenta. A continuación, el área de estudio, la cuenca del río Muga, para pasar posteriormente explicar la metodología. A partir de aquí se presentan los resultados y la discusión, remarcando las similitudes y las divergencias de los resultados obtenidos en este estudio en comparación a los existentes en la literatura científica. A lo que hay que añadir unas conclusiones acompañadas de un conjunto aplicaciones prácticas que se derivan de esta investigación y que pueden favorecer el ahorro hídrico en 
el sector de alojamiento turístico. Finalmente se incluyen un conjunto de consideraciones en relación con las limitaciones propias del estudio desarrollado y las futuras líneas de investigación.

\section{MARCO TEÓRICO}

En los últimos años han proliferado los alojamientos turísticos que apuestan por la implementación de medidas destinadas a reducir su huella ambiental (Mbasera, Du Plessis, Saayman y Kruger, 2016). Para ello es necesario que sean conscientes de las problemáticas actuales para fomentar e implantar, entre otras cosas, medidas de ahorro de agua en sus alojamientos (Kaján \& Saarinen, 2013; Kelly \& Williams, 2007; Olcina Cantos, 2012). A pesar de ello, la voluntad de empresarios y gestores (Hu, Lovelock, Ying, \& Mager, 2019) necesita de la firme colaboración de los huéspedes (Han, Hsu, Lee y Sheu, 2011; Lee, Hsu, Han y Kim, 2010). De este modo, el conocimiento de las prácticas y los hábitos de consumo de agua de los clientes de los alojamientos turísticos resulta de vital importancia para la planificación y el diseño de las estrategias de ahorro de agua que han de desarrollarse en estos alojamientos. Esto es así porque una parte muy importante del consumo de agua de los diversos alojamientos turísticos se lleva a cabo en las habitaciones, más todavía si el alojamiento no cuenta con zonas exteriores ajardinadas o piscinas. Así, incentivar a los clientes a que hagan un uso responsable del agua va a revertir, sin duda, en la disminución de su consumo. En este mismo sentido, está aumentando también la presión sobre los gobiernos y agentes políticos para que contribuyan a fomentar la aplicación de medidas de ahorro e incentiven las prácticas sostenibles y responsables con el medio ambiente en los alojamientos turísticos (Moreo, 2008). Y más aún en un contexto de cambio climático que puede agravar sumamente las problemáticas relacionadas con la disponibilidad de agua y la competencia por este recurso; y provocando además la pérdida de atractivo paisajístico y de turistas en verano, tal como mostraron por ejemplo, Gómez, Armesto, y Cors, (2017) en un estudio llevado a cabo en la comarca del Alt Empordà.

Existen diversas medidas de ahorro de agua que pueden desarrollar los alojamientos turísticos y que han proliferado debido al mayor interés que han ido suscitando la introducción de programas ambientales en la industria de alojamiento turístico (Environmental Management Systems, EMS) (Kasim, 2009). Estudios como los desarrollados por Alonso y Ogle (2010), Bruns-Smith et al. (2015) o Dinarès y Saurí (2015) han concluido que la aplicación de estos programas y de medidas de ahorro de agua como sensores, temporizadores o aireadores en grifos o sistemas de doble descarga en sanitarios son las más usuales debido principalmente al bajo coste de inversión necesario para su implementación y su rápida amortización. Otra de las medidas más implementadas y que favorece el ahorro hídrico consiste en involucrar a los clientes y al personal del propio establecimiento en el uso sostenible de los recursos hídricos, a través por ejemplo de la colocación de carteles informativos (Han y Hyun, 2018b; Page, Essex y Causevic, 2014). Las medidas de ahorro de agua que pueden desarrollar los clientes son también diversas: la reutilización de toallas y sábanas (Kim, Palakurthi y Hancer, 2012a; Gössling, Araña, \& Aguiar-Quintana, 2019), cerrar el grifo al lavarse los dientes o enjabonarse (Gabarda-Mallorquí, Fraguell y Ribas, 2018), el uso del sistema de doble de descarga del sanitario o seguir las indicaciones 
de carteles o informaciones dadas por parte de los alojamientos (Nikolaou, Vitouladitis y Tsagarakis, 2012), entre otras.

En el contexto actual, vista la necesidad de que los turistas se involucren en el ahorro de agua, resulta fundamental conocer qué hábitos de ahorro de agua llevan a cabo durante su estancia y con qué frecuencia lo hacen. Esta necesidad ya fue mostrada por Gilg \& Barr (2006) al afirmar que es fundamental identificar las características de aquellos que llevan a cabo mejores prácticas de ahorro de agua para facilitar a los agentes públicos poder detectar los segmentos sobre los cuales es necesario establecer más presión y saber a quién deben ir dirigidas las medidas que se planteen y diseñen para el mantenimiento y el ahorro de los recursos hídricos. Por esta razón, existen estudios que intentan identificar y analizar, básicamente a través de encuestas a los propios turistas, sus prácticas de ahorro y de consumo de agua cuando están en un alojamiento turístico.

Por ejemplo, los trabajos desarrollados por Gabarda-Mallorquí et al. (2018), en el caso concreto de un hotel de Lloret de Mar (Girona, España), determinan que factores como la edad, el sexo, la procedencia o el nivel de estudios de los turistas son explicativos del grado de concienciación y del comportamiento ambiental de los turistas, y dividen a los clientes en cuatro categorías: concienciados no proactivos, concienciados proactivos, no concienciados no proactivos y no concienciados proactivos. Por su lado, De Urioste-Stone, Scaccia, y Howe-Poteet (2015), concluyen que en Maine (EEUU), concretamente en el Acadia National Park, un destino principalmente de naturaleza, las mujeres son más activas que los hombres cuando se trata de llevar a cabo buenas prácticas ambientales y están más concienciadas sobre los efectos que el cambio climático puede tener sobre la actividad turística y los recursos hídricos. Kim, Palakurthi y Hancer (2012b) mostraron una vez más, en este caso para los hoteles a partir de encuestas online, que el sexo o la edad son factores que determinan el nivel de concienciación y el comportamiento ambiental de los clientes. Muestran como las mujeres tienen mayor disposición que los hombres por alojarse en alojamientos que llevan a cabo buenas prácticas ambientales, al igual que ocurre con el grupo de edad que se sitúa entre los 25 y 34 años si se comparan con los mayores de 55. En otro caso de estudio, a partir de encuestas a turistas que se alojaron en hoteles en Malaysia, Azila et al. (2014) mostraron que cada vez los turistas se muestran más concienciados sobre cuestiones ambientales. Wang et al. (2019) determinaron que el mayor nivel de concienciación sobre problemas ambientales lo tienen los que nombran como motivación principal de su estancia el contacto y el disfrute de la naturaleza, en un caso de estudio concreto situado en Taiwán, concretamente en el Parque Nacional Kenting.

El análisis factorial ha sido, pues, comúnmente utilizado para encontrar relaciones entre los diferentes comportamientos de los turistas, y de este modo se ha visto como el hecho de definir las características de aquellos turistas que llevan a cabo mejores hábitos y prácticas ambientales resulta importante para ayudar a la administración pública a tomar mejores decisiones de gestión y educación, diferenciando los grupos más y menos entusiastas a la hora de llevar a cabo buenos hábitos de ahorro.

Sin duda, el aumento de la concienciación ambiental de la población repercute en prácticas más sostenibles de los turistas, a la vez que estos se sienten más atraídos por aquellos establecimientos que llevan a cabo buenas prácticas ambientales (Han y Hyun, 2018a). En este mismo sentido, Modica, 
Altinay, Farmaki, Gursoy, y Zenga (2020) mostraron que en Cerdeña, después de la realización de 288 encuestas a turistas, si los alojamientos lleven a cabo buenas prácticas ambientales, esto tiene un efecto positivo en los clientes, que se muestran más activos ambientalmente e incluso estarían dispuestos a pagar precios más altos. A pesar de ello, en general, el hecho de que en los alojamientos se lleven a cabo medidas destinadas al ahorro de agua, no es un factor determinante para que el cliente lo escoja. Contrariamente, estudios como el de Kim et al. (2012b), mostraron que existe un segmento turístico que sí que valora ciertas prácticas ambientales, pero se trata más bien de prácticas relacionadas con el ahorro y la eficiencia energética o los residuos y no tan vinculadas propiamente el ahorro de agua.

En definitiva, si bien en los últimos años ha aumentado la concienciación de la población entorno a la necesidad de hacer un uso cada vez más eficiente del agua, todavía hoy existe una incoherencia entre su nivel de concienciación y las medidas de ahorro que llevan a cabo, más aún cuando se encuentran en un alojamiento turístico (Han y Hyun, 2018a; Deyà y Tirado, 2011). Esta es la razón que esgrimen algunos responsables de los alojamientos para no implementar medidas de ahorro de agua, ya que se muestran escépticos sobre la voluntad de los clientes a hacer un uso responsable del agua, especialmente en aquellos alojamientos de mayor categoría (Jarvis y Ortega, 2010; Leslie, 2001; Warren, Becken, y Coghlan, 2017). Los turistas deben situarse, pues, en el punto de mira de las estrategias de las administraciones y empresas turísticas cuando se trata de fomentar el ahorro hídrico en el sector turístico y contribuir a la sostenibilidad de las destinaciones (Ayuso, 2007). Sin su participación, las medidas que implementen los alojamientos pueden perder efectividad.

El objetivo principal de este artículo es analizar el comportamiento de los clientes de alojamientos turísticos respecto al consumo de agua en la cuenca del río Muga (Girona). Una cuenca donde se prevén futuros problemas de escasez de agua vinculados a un aumento de su demanda por el incremento de turistas y también como consecuencia directa de los efectos del cambio climático (Pascual et al., 2016).

\section{Área de ESTUdio}

La cuenca del río Muga se encuentra en el extremo noreste de la Península Ibérica (Figura 1), comprende 52 municipios y un área aproximada de $1150 \mathrm{~km}^{2}$. Se trata de una destinación turística donde se augura una clara disminución de los recursos hídricos como consecuencia directa de los efectos del cambio climático y el aumento de la demanda por parte de una población en aumento y unas actividades económicas que requieren cada vez más agua para su sustento y desarrollo (agricultura y turismo, principalmente) (Oficina Catalana del Canvi Climàtic, 2013; Pascual et al., 2016; Pla et al., 2017; Vicente-Serrano et al., 2016).

Una de estas actividades económicas claramente al alza es el turismo. En el año 2018 visitaron la comarca del Alt Empordà aproximadamente 1.200.000 personas, lo que ha representado más de 4.000.000 de pernoctaciones (Diputació de Girona, 2019). Los alojamientos turísticos presentes en la zona son 117 hoteles, 14 campings y 86 casas de turismo rural y en conjunto ofrecen 23.000 plazas de alojamiento. Se trata, pues, de un importante destino en el que se distinguen tres zonas bien diferenciadas: zona litoral, zona urbana y zona interior o rural. En el litoral predomina un turismo de sol y playa, 
mientras que en el interior encontramos básicamente un turismo de naturaleza. En las zonas urbanas de Figueres y La Jonquera domina un turismo más cultural, de negocios o comercial. La Jonquera, municipio fronterizo con Francia, se ha convertido en un importante foco de turismo comercial y Figueres, con la sede del museo Dalí y a la vez capital comarcal, juega un importante rol especialmente de turismo cultural y también de negocios y comercial.

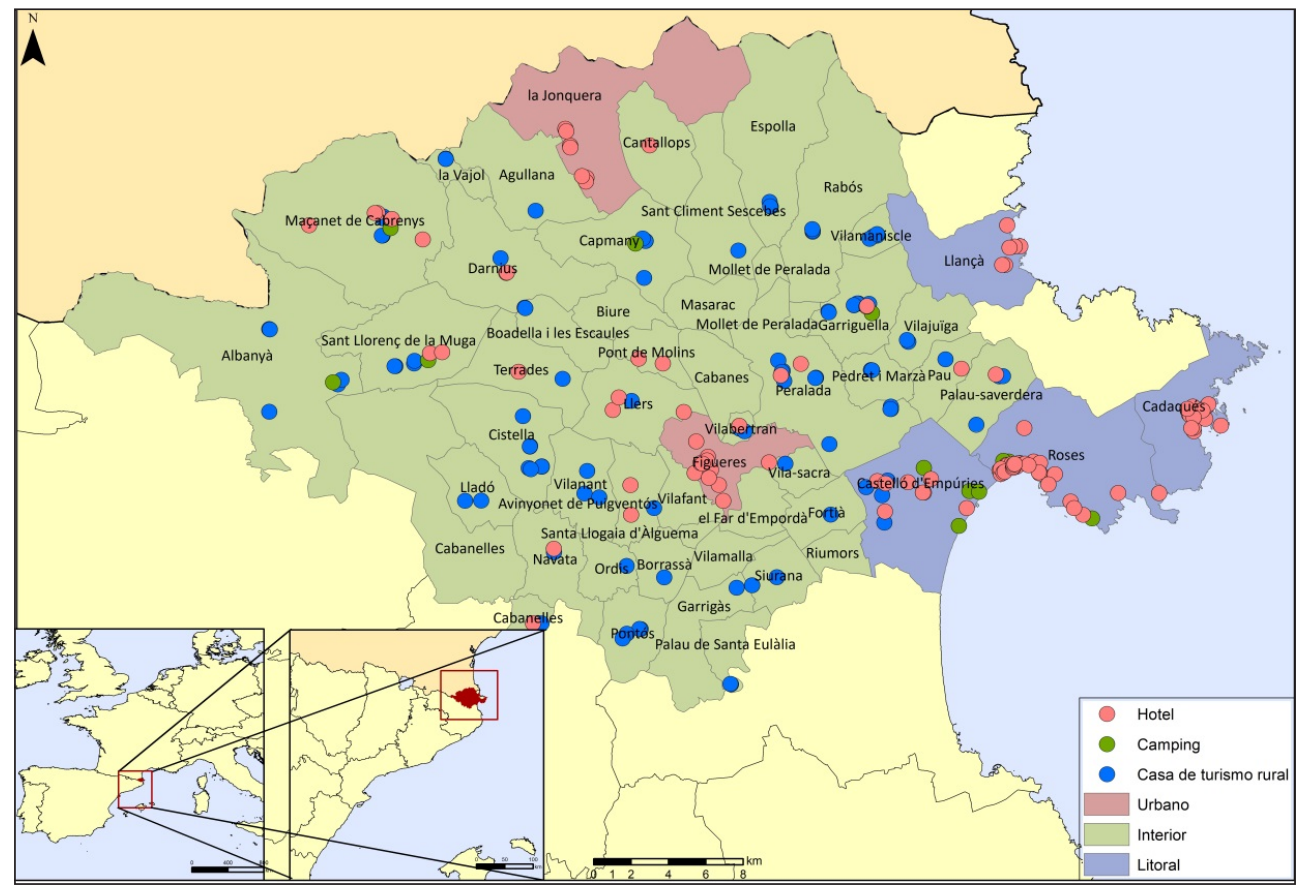

Figura 1: Localización del área de estudio. Fuente: elaboración propia a partir de los datos de la Guía de establecimientos turísticos (Generalitat de Catalunya, 2017)

Se trata de una zona que históricamente ya ha sufrido los efectos derivados de la falta de disponibilidad de agua lo que ha llevado a diversos episodios de conflictos entre los sectores que precisan de este recurso. Estamos hablando, por ejemplo, de la agricultura, el turismo, el consumo doméstico o el mantenimiento de los caudales naturales del río y la conservación de las zonas de humedales. Ventura, Ribas y Saurí (2000) identificaron 22 episodios conflictivos por el uso del agua en la cuenca del río Muga entre los años 1980 y 1999. Uno de los episodios más conflictivos fue el que sucedió en el año 1984 cuando el agua del embalse de Darnius-Boadella disminuyó hasta el 25\% de sus reservas y creó una importante alarma social, episodio conocido como "la guerra de los pozos" (Gabarda, Ribas y Daunis-i-Estadella, 2015); o bien en el año 1998 cuando el embalse llegó al 8,75\% de su capacidad (Ventura et al., 2000). 
El último conflicto destacado ha tenido lugar entre los años 2007 y 2008, con un episodio de sequía muy prolongado en el tiempo, el más importante de los últimos 70 años (Pla et al., 2017; Ribas y Saurí, 2010). Fue el 5 de octubre de 2007 cuando entró en vigor el Decreto de Sequía (Decreto-Ley 9/2017, por el cual se adoptan medidas urgentes para paliar los efectos producidos por la sequía en determinadas cuencas hidrográficas (BOE 240, de 6.10.2017)) para hacer frente a una de las más intensas sequías acaecidas en Cataluña. Este periodo se prolongó hasta prácticamente principios de año 2009, cuando la última cuenca salió del estado de excepcionalidad (precisamente, la cuenca del río Muga) después de un período de más de un año sin precipitaciones abundantes en la cabecera del río, que se tradujo en una constante disminución de los niveles de agua almacenada en el embalse y su consecuente alarma social (El País, 2007 (17 de julio) y 2008 (5 de junio); El Periódico, 2008 (23 de junio)). En este periodo se llegó a niveles cercanos al 20\% de la capacidad del embalse (El Pais, 2008 (29 de diciembre)), lo que casi se tradujo en el corte de suministro a la población.

\section{Metodología}

Para responder al objetivo principal de este estudio se ha procedido a la realización de encuestas a turistas que se hospedan en alojamientos turísticos de la cuenca del río Muga. El modelo de encuesta que se diseñó tenía dos requisitos técnicos fundamentales: que fuese rápida de contestar (menos de 10 minutos) y que incluyera las preguntas oportunas para dar respuesta a los objetivos planteados. La encuesta fue validada por un grupo de expertos perteneciente al Grupo de Investigación Agua, Territorio, Turismo y Sostenibilidad (GRATTS) vinculados a la Universidad de Girona.

Los alojamientos seleccionados para realizar las encuestas fueron, en parte, los mismos cuyos responsables habían sido previamente entrevistados, ya que de esta manera se disponía de información detallada de las características de estos alojamientos. Para determinar la muestra de persones a encuestar, se calculó el número de turistas totales de la destinación. Al no disponer de los datos específicos a nivel municipal, se realizó una estimación a partir de los turistas totales de la comarca del Alt Empordà (que se corresponde en buena parte con el área de estudio) y el número de plazas de alojamiento existentes en campings, casas de turismo rural y hoteles. Estos cálculos reportaron que la zona de estudio recibe aproximadamente 650.000 turistas anuales, lo que se traduce en más de dos millones de pernoctaciones anuales para un total de, aproximadamente, 23.000 plazas de alojamiento repartidas en 117 en hoteles, 14 campings y 86 casas de turismo rural (Diputació de Girona, 2017). El muestreo aleatorio simple determinó la necesidad de realizar un mínimo de 384 encuestas. Las encuestas se realizaron en las zonas comunes de estos establecimientos. Teniendo en cuenta que era necesario diversificar la muestra y tener respuestas de las diferentes tipologías, categorías y localizaciones, se realizaron encuestas en 19 alojamientos durante los meses de mayor afluencia turística (junio-setiembre). Una vez finalizadas, se validaron un total de 580 encuestas.

Las encuestas se estructuraron en cuatro bloques: 1) perfil del encuestado; 2) valoración de los elementos que requieren agua; 3) prácticas propias y consumo de agua; y, 4) percepción sobre el cambio 
climático y sus efectos. En este estudio se utilizan las respuestas correspondientes a las preguntas de los bloques 1,3 y 4 :

a) Para conocer y analizar los hábitos de consumo y ahorro de agua de los turistas se analizaron las respuestas de los turistas a las siguientes afirmaciones pertenecientes al bloque tres:

Cierro el grifo mientras me enjabono

Cierro el grifo mientras me lavo los dientes

Hago un uso racional del agua

Utilizo adecuadamente el sistema de doble descarga del sanitario

Se les preguntó la frecuencia con la que llevaban a cabo estos hábitos durante su estancia en una escala Likert de 1 a 5: 1, nunca; 2, raramente; 3, a veces; 4, casi siempre y 5, siempre.

b) Para conocer su nivel de concienciación en relación con la disponibilidad de agua, se les preguntó por su nivel de acuerdo o desacuerdo en relación a las dos siguientes afirmaciones del bloque 4:

En Cataluña hay suficiente agua, no hemos de preocuparnos en ahorrarla

No creo en el cambio climático y no pienso que tendremos menos agua, por lo cual no es necesario establecer medidas por este motivo

Estas cuestiones fueron respondidas también en una escala Likert de 1 a $5: 1$, totalmente en desacuerdo; 2 , en desacuerdo; 3 , ni en acuerdo ni en desacuerdo; 4, de acuerdo y 5 , totalmente de acuerdo.

Con los resultados de estas preguntas, y gracias al soporte del software SPSS (v.23) se realizó un análisis clúster bietápico, que sugirió la presencia de tres grupos bien diferenciados en función de cómo los encuestados perciben las afectaciones y el grado de implicación en llevar a cabo buenas prácticas en el uso del agua. Unos resultados que vienen reforzados por un índice de fiabilidad bueno, tal como demostraron los resultados del análisis clúster realizado (calidad del clúster $>0,5$ )

Finalmente, se analizaron las características principales de los diferentes clústeres y se definieron diversas estrategias para aumentar la concienciación y los buenos hábitos en el uso del agua por parte de los turistas.

\section{Resultados}

En este apartado se muestran los resultados que se desprenden de la realización de las encuestas a los clientes de alojamientos turísticos de la zona de estudio. Antes de entrar a describir los resultados se hace necesario explicar brevemente la composición sociodemográfica de la muestra de turistas encuestados. Como puede observarse en la tabla 1 , predominan las mujeres $(53,4 \%)$ frente a los hombres $(46,6 \%)$, y turistas de edades comprendidas entre los 26 y 40 años (38,9\%) y los 41 y 55 (32,3\%). Por lo que se refiere al nivel de estudios alcanzados, los encuestados con estudios universitarios son los más numerosos, con poco más del $42 \%$, seguido por el 24,1\% que tiene estudios de formación profesional; siendo los turistas con estudios primarios o sin estudios los menos frecuentes. La mayor parte procede de países europeos (exceptuando España, 50,1\%), de los cuales destacan los procedentes de Francia, 
debido a la proximidad geográfica entre ambos países. En segunda posición casi el $23 \%$ del total de turistas encuestados procede de Barcelona y su área metropolitana. Finalmente, teniendo en cuenta la motivación principal de la estancia, predomina el turismo de sol y playa $(45,3 \%)$ y de naturaleza $(28,7 \%)$, seguido por el cultural y urbano $(11,5 \%)$ o los deportes $(7,3 \%)$.

Tabla 1: Características sociodemográficas de la muestra de turistas encuestados. Fuente: elaboración propia a partir de los datos recogidos en las encuestas

\begin{tabular}{|c|c|c|}
\hline & & $\%$ total \\
\hline \multirow{5}{*}{ Edad } & $<25$ & 13,7 \\
\hline & $26-40$ & 38,9 \\
\hline & $41-55$ & 32,3 \\
\hline & $56-65$ & 11,9 \\
\hline & $>65$ & 3,2 \\
\hline \multirow{2}{*}{ Sexo } & Masculino & 46,6 \\
\hline & Femenino & 53,4 \\
\hline \multirow{6}{*}{ Formación académica completada } & Sin estudios & 0,5 \\
\hline & Estudios primarios & 4,9 \\
\hline & Estudios secundarios & 15,0 \\
\hline & Bachillerato & 13,8 \\
\hline & Estudios de formación profesional & 24,1 \\
\hline & Estudios universitarios & 42,2 \\
\hline \multirow{6}{*}{ Procedencia } & Comarcas gerundenses & 7,6 \\
\hline & Área metropolitana de Barcelona & 22,8 \\
\hline & Resto de Cataluña & 6,7 \\
\hline & Resto de España & 10,2 \\
\hline & Resto de Europa & 50,5 \\
\hline & Resto del mundo & 2,2 \\
\hline \multirow{7}{*}{ Motivación principal } & Negocios & 2,6 \\
\hline & Deportes & 7,3 \\
\hline & Naturaleza & 28,7 \\
\hline & Sol y playa & 45,3 \\
\hline & Cultural y/o urbano & 11,5 \\
\hline & Eventos & 2,3 \\
\hline & Juvenil y/o escolar & 2,3 \\
\hline
\end{tabular}


Los datos de interés que se extraen de las encuestas para la realización de este estudio se derivan de los bloques 3 y 4 de la misma, junto con los datos relativos al perfil sociodemográfico de los clientes.

Los resultados de las encuestas muestran comportamientos dispares entre los clientes en relación con las prácticas de ahorro y uso del agua en los alojamientos, al mismo tiempo que diferente nivel de concienciación sobre las problemáticas relacionadas con el cambio climático y en especial sobre la disponibilidad de agua. A partir del análisis de frecuencia de respuestas de cada una de las preguntas se ha realizado un análisis clúster, cuyos resultados son los que se muestran en la tabla 2.

Tabla 2: Clasificación de los clientes en cada uno de los clústeres. Fuente: elaboración propia a partir de datos recogidos en las encuestas

\begin{tabular}{|c|c|c|c|c|c|}
\hline Afirmación planteada & Frecuencia/nivel de acuerdo & Clúster 1 & Clúster 2 & Clúster 3 & $\%$ total \\
\hline \multirow{5}{*}{$\begin{array}{l}\text { Cierro el grifo mientras me } \\
\text { enjabono }\end{array}$} & Nunca & 52 & 6 & 4 & 11 \\
\hline & Raramente & 26 & 4 & 2 & 6 \\
\hline & A veces & 30 & 33 & 0 & 11 \\
\hline & Casi siempre & 25 & 87 & 0 & 19 \\
\hline & Siempre & 12 & 62 & 237 & 54 \\
\hline \multirow{5}{*}{$\begin{array}{l}\text { Cierro el grifo mientras me } \\
\text { lavo los dientes }\end{array}$} & Nunca & 30 & 0 & 1 & 5 \\
\hline & Raramente & 9 & 0 & 1 & 2 \\
\hline & A veces & 25 & 17 & 1 & 7 \\
\hline & Casi siempre & 32 & 67 & 3 & 18 \\
\hline & Siempre & 49 & 108 & 237 & 68 \\
\hline \multirow{5}{*}{$\begin{array}{l}\text { Hago un uso racional del } \\
\text { agua }\end{array}$} & Nunca & 26 & 0 & 1 & 5 \\
\hline & Raramente & 26 & 2 & 2 & 5 \\
\hline & A veces & 52 & 39 & 11 & 18 \\
\hline & Casi siempre & 31 & 96 & 10 & 24 \\
\hline & Siempre & 10 & 55 & 219 & 49 \\
\hline \multirow{5}{*}{$\begin{array}{l}\text { Uso adecuadamente el } \\
\text { sistema de doble descarga } \\
\text { del sanitario }\end{array}$} & Nunca & 41 & 11 & 15 & 12 \\
\hline & Raramente & 13 & 6 & 4 & 4 \\
\hline & A veces & 47 & 32 & 16 & 16 \\
\hline & Casi siempre & 17 & 81 & 7 & 18 \\
\hline & Siempre & 27 & 62 & 201 & 50 \\
\hline \multirow{5}{*}{$\begin{array}{l}\text { En Cataluña hay suficiente } \\
\text { agua, no hemos de } \\
\text { preocuparnos por ahorrarla }\end{array}$} & Totalmente de acuerdo & 30 & 137 & 162 & 57 \\
\hline & De acuerdo & 36 & 47 & 35 & 20 \\
\hline & Ni de acuerdo ni en desacuerdo & 62 & 6 & 31 & 17 \\
\hline & En desacuerdo & 10 & 2 & 5 & 3 \\
\hline & Totalmente en desacuerdo & 7 & 0 & 10 & 3 \\
\hline
\end{tabular}




\begin{tabular}{llcccc}
\hline No creo en el cambio & Totalmente de acuerdo & 62 & 158 & 201 & 73 \\
\cline { 2 - 6 } $\begin{array}{l}\text { climático y no pienso que } \\
\text { tendremos menos agua, } \\
\text { por lo cual no es necesario }\end{array}$ & De acuerdo & 32 & 28 & 12 & 12 \\
\cline { 2 - 6 } $\begin{array}{l}\text { establecer medidas por este } \\
\text { motivo }\end{array}$ & Ni de acuerdo ni en desacuerdo & 37 & 1 & 11 & 8 \\
\cline { 2 - 6 } & En desacuerdo & 7 & 3 & 11 & 4 \\
\hline
\end{tabular}

En este caso concreto, los resultados que se extraen del análisis (Tabla 2), muestran la existencia de tres grupos de turistas claramente diferenciados (Figura 2).

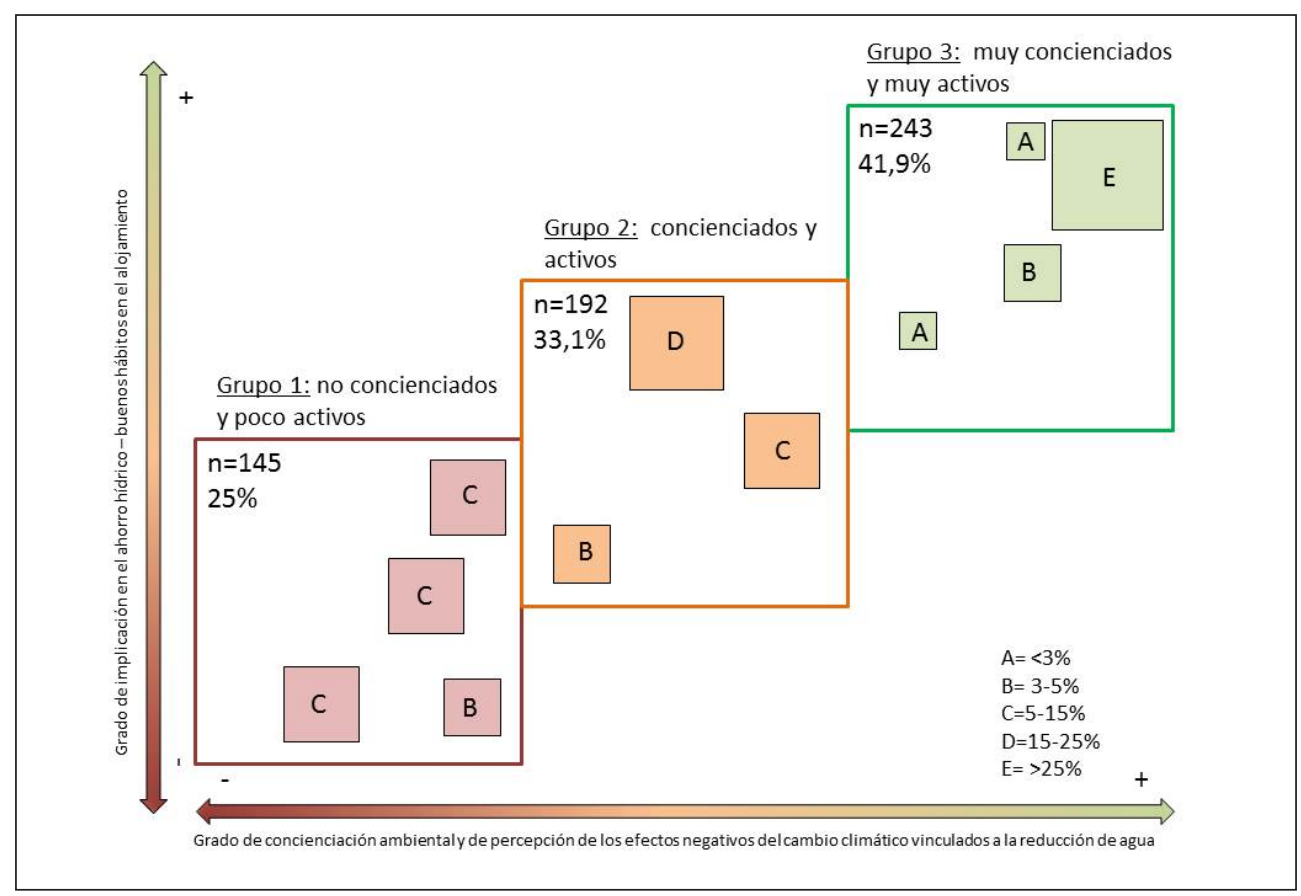

Figura 2: Clasificación de los perfiles de turistas encuestados y \% de encuestados en cada uno de los clústeres. Fuente: elaboración propia a partir de datos recogidos en las encuestas.

En primer lugar, el Grupo 1 ( $25 \%$ de los encuestados) es el menos concienciado entorno a los problemas de escasez hídrica derivados del cambio climático y la disponibilidad de agua en Cataluña. Aquí se encuentran los turistas que menos llevan a cabo buenas prácticas de ahorro hídrico, de tal manera que pueden ser considerados como "poco activos medioambientalmente". En cuanto a su origen, se trata mayoritariamente de turistas europeos ( $56 \%$ del total, básicamente franceses o de fuera de países mediterráneos) y procedentes de Barcelona y alrededores (25\%). Por edades dominan los de franjas 
de edades comprendidas entre los 26-40 años (35\%) y los 41-55 (27\%). Destaca que en este grupo se encuentra la mayor proporción de turistas con estudios primarios (6\%) y Bachillerato (20\%) en comparación con los demás grupos. También este clúster reúne la mayor proporción de turistas alojados en hoteles (34\%) y la menor de turistas en casas de turismo rural (2\%) en comparación con los demás grupos. Por lo que se refiere a la localización de los alojamientos, el 56,6\% los encontramos en el litoral y así mismo es el grupo que contiene más proporción de turistas alojados en hoteles urbanos. Por otro lado, si nos centramos en la motivación principal de la estancia, en este grupo se encuentra la menor proporción de turistas que mencionan el turismo de naturaleza en relación con los demás grupos.

El Grupo 2 (33,1\% de los encuestados) está formado por aquellos turistas que en general llevan a cabo buenas prácticas, y su nivel de percepción de los problemas de disponibilidad de agua es mayor que en el grupo 1. Está formado por personas concienciadas y activas medioambientalmente. Se trata de turistas cuyas respuestas se encuentran entre el 2 y el 3 en la escala de Likert en las preguntas relacionadas con el cambio climático y la disponibilidad de agua en Cataluña. Se considera que están mínimamente concienciados sobre los problemas de escasez de agua y llevan a cabo algunas veces o casi siempre prácticas de ahorro hídrico ( 3 y 4 de la escala Likert). Este segundo grupo está formado mayoritariamente por turistas de edades comprendidas entre los 26 y 40 años (41\%) y con estudios universitarios (42\%) o de formación profesional (23\%). El 52\% de los turistas de este grupo son europeos y predominan los que se alojan en campings (64\%), si bien en este grupo se encuentra la mayor proporción de turistas alojados en casas de turismo rural (8\%). Referente a la localización, predominan los turistas alojados en establecimientos ubicados en el litoral (62,5\%), seguido por los que se alojan en establecimientos de interior $(33,3 \%)$. En lo referente a la motivación de la estancia, en este segundo grupo se encuentra la mayor proporción de turistas que mencionan las actividades relacionadas con el sol y playa que en los demás grupos (46\%).

Finalmente, el Grupo 3 es el más numeroso y está integrado por aquellos turistas conocedores de los problemas de disponibilidad hídrica existentes en Cataluña y que consideran que el cambio climático agravará aún más esta problemática. Se trata, además, de los que con mayor frecuencia llevan a cabo prácticas de ahorro de agua (41,9\% del total). Son, pues, turistas altamente concienciados entorno a los problemas de escasez hídrica, por lo que no es de extrañar que más del $80 \%$ de los encuestados de este grupo estén totalmente en desacuerdo o en desacuerdo con la afirmación que en Cataluña hay suficiente agua, y que considera que el cambio climático comportará aún mayores problemas de falta de agua. Predominan los que tienen edades comprendidas entre 26-40 años (37\%) y 41-55 (35\%), si bien es de destacar que reúne también la mayor proporción de personas mayores de 65 años. El 45\% de estos encuestados disponen de estudios universitarios y el $26 \%$ estudios de formación profesional, siendo el grupo que reúne un mayor número de encuestados de elevado nivel formativo. Cabe señalar que se trata del grupo con mayor proporción de turistas alojados en campings (73\%) y menor en hoteles (26\%). Si tenemos en cuenta la localización de estos turistas, es importante destacar que se trata del grupo con un mayor porcentaje de turistas alojados en establecimientos de interior (35,2\%). Finalmente, se trata del grupo que contiene mayor proporción de clientes de alojamientos turísticos que están en el 
destino por el disfrute y las actividades relacionadas con la naturaleza y el entorno (33\%) y menor en las actividades relacionadas con el turismo urbano y/o cultural (9\%).

\section{Discusión}

Este estudio pone de manifiesto que existen diferentes perfiles de turistas en relación con las buenas prácticas de consumo de agua y su nivel de concienciación ambiental frente a los problemas del agua.

En general, se puede decir que, a mayor concienciación ambiental, mayor es la frecuencia con la que se desarrollan buenos hábitos de consumo de agua. Ambos factores siguen una correlación lineal positiva. A pesar de esta primera consideración general, pero, existen algunos casos en los que no necesariamente el hecho de tener una buena concienciación ambiental resulta en llevar a cabo buenas prácticas ambientales, tal como mostraron ya Gabarda-Mallorquí et al. (2018). Se ve este resultado en algunos de los encuestados que, a pesar de mostrar un grado de acuerdo elevado en relación con las preguntas relacionadas con el cambio climático y la disponibilidad de agua, no siempre realizan prácticas de ahorro de agua durante su estancia. En este sentido, se ha visto como por ejemplo los clientes de forma general muestran un conocimiento alto de que el cambio climático puede afectar a la zona y la disponibilidad de agua. A pesar de ello, algunos de los encuestados afirman que cuando están en un alojamiento turístico consumen más agua que cuando se encuentran en sus residencias habituales, un resultado que ya fue también mencionado en el estudio de Deyà y Tirado (2011) en el caso de la isla de Mallorca.

A parte de esto, si nos fijamos en qué características de los propios clientes determinan que estos se ubiquen en uno u otro grupo, se ve como el sexo, la edad, la motivación de la estancia o el nivel de estudios se muestran como factores importantes a la hora de definirlos. Aun así, este estudio, al contrario de lo que concluyen De Urioste-Stone et al. (2015) y Aprile y Fiorillo (2017), no ha podido demostrar que el sexo sea significativamente importante a la hora de diferenciar comportamientos ya que es indiferente el sexo de los clientes a la hora de situarlos en uno u otro grupo. En concordancia con nuestro estudio, Lam (2006) o anteriormente, Roberts (1996) ya determinaron en sus respectivos estudios que el sexo no es un factor determinante y que hombres y mujeres tienen unos comportamientos similares.

Por otro lado, en los tres grupos existe una cierta homogeneidad de edades, aunque la mayor proporción de mayores de 65 años se sitúa en el grupo que realiza mejores prácticas y está más concienciado ambientalmente, tal como demostraron también Gabarda-Mallorquí et al. (2018) para el caso de estudio de un hotel de Lloret de Mar. Por tanto, aunque no puede concluirse que la edad influya de manera significativa en el nivel de concienciación ambiental en relación con las buenas prácticas de consumo de agua, sí que la mayor proporción de mayores de 65 años se encuentra en el grupo 3. Otros estudios, como los realizados por Clark y Finley (2007), Gilg y Barr (2006) o Gregory y Leo (2003), también mostraron como los que tienen edades más avanzadas tienden a hacer un uso más responsable de los recursos lo que se relaciona directamente con el hecho que posiblemente los de edades más avanzadas han padecido episodios anteriores de precariedad y escasez hídrica. 
En referencia a la motivación de la estancia, la mayor proporción de encuestados que mencionan el contacto y disfrute de la naturaleza como su motivación principal se encuentran en el grupo 3, siendo en el grupo 1 y 2 las mayores proporciones de aquellos que mencionaron los negocios o el turismo relacionado con el sol y la playa. Este resultado difiere de los resultados obtenidos por Millar, Mayer y Baloglu (2012) que muestran como los turistas de negocios muestran hábitos más sostenibles y mejor conciencia que aquellos que viajan por ocio. A pesar de ello, pero, conviene matizar que se trata de un estudio llevado a cabo en el caso de los hoteles y no en campings y casas de turismo rural, los clientes de cuyos alojamientos presentan unos hábitos de ahorro de agua con mayor frecuencia en nuestro caso de estudio.

La mayor proporción de campistas se encuentra en el grupo 3, lo que nos indica una mayor concienciación y proactividad medioambiental de los clientes alojados en esta tipología de establecimientos. Un comportamiento que se explicaría seguramente porque valoran más el contacto con la naturaleza, y, por ende, están más preocupados por los problemas que se pueden derivar de la disminución de la disponibilidad hídrica.

Finalmente, el nivel de estudios se muestra también como un elemento para tener en cuenta. Así, al contrario de lo que proponen Aprile y Fiorillo (2017) sí que puede decirse que, al menos en nuestro caso, el nivel de estudios y el nivel de concienciación y la proactividad ambiental de los turistas están positivamente relacionados. Los resultados muestran, por ejemplo, que los clientes con niveles formativos más altos se encuentran en mayor proporción en los grupos 2 y 3. Contrariamente, los que presentan niveles formativos inferiores se encuentran en mayor proporción en el grupo 1. Este resultado coincide con el obtenido por Gabarda-Mallorquí et al., (2018) pero difiere de los obtenidos por Gregory y Leo (2003).

\section{Conclusiones}

Existen diferentes perfiles de turistas atendiendo a sus hábitos y comportamientos en relación con el consumo de agua y su nivel de concienciación entorno a la problemática del agua. En este estudio realizado para el caso de estudio de la cuenca del río Muga se han identificado tres perfiles claramente diferenciados: no concienciados y poco activos ambientalmente; concienciados y activos; y, muy concienciados muy activos.

Se ha podido comprobar como existe un número elevado de turistas (25\%) que no son conscientes del potencial impacto del cambio climático en la disponibilidad hídrica, ni tampoco en relación con los problemas actuales en la zona de estudio, y como consecuencia no llevan a cabo buenas prácticas de ahorro de agua. Por este motivo, resulta del todo imprescindible focalizar acciones de sensibilización en este segmento turístico.

Ello será posible a partir de la determinación y la firme voluntad de todos los agentes involucrados en la actividad turística, que comprende, además de turistas, a los responsables de alojamientos turísticos y a las administraciones públicas. Finalmente cabe destacar que afortunadamente el grupo de turistas más numeroso es aquel que sí declara estar concienciado ambientalmente y que afirma que 
lleva a cabo buenas prácticas y hábitos en el uso del agua, formado por poco más del $40 \%$ del total de encuestados.

En definitiva, y a la luz de los resultados obtenidos en este estudio, las características sociodemográficas, la localización y el tipo de alojamiento deben ser tenidas en cuenta a la hora de plantear las mejores estrategias posibles destinadas a conseguir un uso más eficiente del agua. Los resultados de este estudio apuntan a que debe focalizarse de forma especial la atención en aquellos turistas con menor nivel educativo, jóvenes y que se alojan, preferentemente, en hoteles, de localizaciones urbanas y de litoral.

\section{Aplicaciones prácticas de la inVestigación}

Los resultados de esta investigación nos llevan a presentar algunas de las estrategias que podrían desarrollarse para aumentar la concienciación general de los turistas sobre los problemas relacionados con los recursos hídricos, en la cuenca mediterránea y de forma especial en el área de estudio.

En primer lugar, visto que entre aquellos que llevan a cabo hábitos de ahorro de agua con menor frecuencia (grupo 1) predominan aquellos que provienen de países no-mediterráneos, sería conveniente que desde sus países de origen se les informe sobre las problemáticas existentes con el agua en el país de destino. Por ejemplo, en medios de transporte como el tren o el avión podrían incluirse videos y/o carteles informativos. Un caso práctico concreto y con gran repercusión fue el que se desarrolló por parte del gobierno balear durante la campaña de verano de 2018, en el cual se informaba a los visitantes durante su llegada al aeropuerto de las diferencias pluviométricas existentes entre las Islas Baleares y sus países de origen. Por tanto, ya desde su llegada al aeropuerto los turistas eran conscientes que se encontraban en una destinación que por sus características naturales -se trata de una isla mediterránea, y por tanto sus recursos hídricos son limitados- tenía una disponibilidad de agua menor que en sus lugares de origen.

Por otro lado, también se hace imprescindible que los alojamientos insten a los clientes a ahorrar agua. Por ejemplo, carteles en los baños o recomendaciones para reutilizar toallas más de un día, una estrategia que ya se encuentra implementada en buena parte de los alojamientos de nuestro entorno pero que requiere aún de una mayor insistencia al respecto y de un estricto cumplimiento por parte de los mismos alojamientos turísticos. Otra medida que podría implementarse sería facilitar a los clientes información referente a su consumo de agua durante la estancia a fin de animarlos a reducir este consumo. Sí que es verdad que esta interesante iniciativa requiere de una inversión económica por parte de los alojamientos, quienes deberían instalar contadores individualizados y no siempre disponen de la capacidad o la voluntad inversora para su instalación. Adicionalmente, este monitoreo podría utilizarse para la implementación de descuentos en el precio de la estancia o bonificaciones en algunos servicios del alojamiento a aquellos clientes que realizan un uso más eficiente del agua. Cumpliendo, así, con un doble objetivo: la reducción del consumo de agua, por tanto, ahorro de costes operativos por parte del alojamiento, y seguramente, una mayor satisfacción de la clientela por los beneficios que le reportan estos incentivos. Finalmente, los responsables de los alojamientos deberían asegurarse de que todas las 
informaciones y programas de ahorro de agua que llevan a cabo son debidamente explicados a los clientes a través de las pertinentes campañas informativas en los propios alojamientos, pero también en sus páginas web y demás plataformas donde se publicitan, lo que puede ayudar a atraer a aquellos turistas que valoran que existan este tipo de medidas ambientales en los alojamientos turísticos.

\section{LIMITACIONES Y FUTURAS LÍNEAS DE INVESTIGACIÓN}

Al llevar a cabo esta investigación se han constatado algunas limitaciones y al mismo tiempo se han detectado nuevas líneas potenciales de investigación.

En primer lugar, la muestra de turistas encuestados, aunque es representativa, incorpora un sesgo en relación con la tipología de alojamientos dónde se han realizado las encuestas. En campings y en hoteles, las facilidades para encontrar a los clientes y poder hacer las encuestas han sido mayores que en casas de turismo rural, que generalmente cuentan con menos plazas y por tanto cada vez que se accedió al alojamiento pudieron realizarse pocas encuestas. Por tanto, en futuras investigaciones deberá aumentarse la representatividad de las casas de turismo rural, del mismo modo que deberán añadirse otras tipologías de alojamientos como los apartamentos turísticos, que, si bien en este estudio no han sido tenidos en cuenta, están teniendo un auge importante en los últimos años en la zona de estudio. Así mismo, por causas de la temporalidad de la investigación, las encuestas se llevaron a cabo durante los meses de verano, por lo que se propone que debieran llevarse a cabo encuestas durante otras épocas del año, captando así, otros perfiles de turistas, para tener un análisis más en profundidad de la realidad de las destinaciones turísticas.

Por otro lado, este estudio se llevó a cabo antes de que se sucedieran dos importantes fenómenos que sin duda están teniendo una influencia crucial en la actividad turística: el temporal Gloria, sucedido en enero de 2020 y la pandemia provocada por el COVID-19. Estos dos fenómenos pueden suponer un cambio en las tendencias de consumo, por lo que deberán contrastarse los resultados obtenidos en esta investigación con los que se sucedan de otros estudios posteriores para detectar divergencias significativas después de eventos como los referidos anteriormente. Todo ello debe tenerse en cuenta, no sin olvidar, pero, que existen otras crisis estructurales como el cambio climático o la disminución de recursos naturales que pueden tener una incidencia mucho más severa en la actividad turística (Gössling, Scott, \& Hall, 2020).

\section{Agradecimientos}

Este artículo reúne parte de los resultados del proyecto de investigación "Incentivos y barreras para el ahorro hídrico en el sector turístico. Análisis y propuestas para una gestión eficiente de agua”, financiado por el MINECO (referencia CSO2016-75740-P). 


\section{ReFERENCIAS}

Alonso, Abel D., \& Ogle, A. (2010): Tourism and hospitality small and medium enterprises and environmental sustainability. Management Research Review, 33(8), 818-826. https://doi. org/10.1108/01409171011065626.

Alonso, Abel Duarte. (2008): How Australian Hospitality Operations View Water Consumption and Water Conservation: An Exploratory Study. Journal of Hospitality Eु Leisure Marketing, 17(3-4), 354-372. https://doi.org/10.1080/10507050801984917.

Aprile, M. C., \& Fiorillo, D. (2017): Water conservation behavior and environmental concerns: Evidence from a representative sample of Italian individuals. Journal of Cleaner Production, 159, 119-129. https://doi.org/10.1016/j.jclepro.2017.05.036.

Ayuso, S. (2007): Comparing voluntary policy instruments for sustainable tourism: The experience of the Spanish hotel sector. Journal of Sustainable Tourism, 15(2), 144-159. https://doi.org/10.2167/ jost617.0.

Azila, N., Noor, M., \& Kumar, D. (2014): ECO Friendly 'Activities ' VS ECO Friendly 'Attitude ': Travelers Intention to Choose Green Hotels in Malaysia, 30(4), 506-513. https://doi.org/10.5829/ idosi.wasj.2014.30.04.14062.

Bohdanowicz, P. (2006): Environmental awareness and initiatives in the Swedish and Polish hotel industries-survey results. International Journal of Hospitality Management, 25, 662-682. https://doi. org/10.1016/j.ijhm.2005.06.006.

Bruns-Smith, A., Choy, V., Chong, H., \& Verma, R. (2015): Environmental Sustainability in the Hospitality Industry: Best Practices, Guest Participation, and Customer Satisfaction Part of the Hospitality Administration and Management Commons.

Clark, W. A., \& Finley, J. C. (2007): Determinants of Water Conservation Intention in Blagoevgrad, Bulgaria. Society $\mathcal{E}$ Natural Resources, 20(7), 613-627. https://doi.org/10.1080/08941920701216552.

De Urioste-Stone, S. M., Scaccia, M. D., \& Howe-Poteet, D. (2015): Exploring visitor perceptions of the influence of climate change on tourism at Acadia National Park, Maine. Journal of Outdoor Recreation and Tourism, 11,34-43. https://doi.org/10.1016/j.jort.2015.07.001.

Deyà, B., \& Tirado, D. (2011): Hotel water consumption at a seasonal mass tourist destination. The case of the island of Mallorca. Journal of Environmental Management, 92(10), 2568-2579. https:// doi.org/10.1016/j.jenvman.2011.05.024.

Dinarès, M., \& Saurí, D. (2015): Water consumption patterns of hotels and their response to droughts and public concerns regarding water conservation: The case of the Barcelona hotel industry during the 2007-2008 episode. Documents d'anàlisi Geogràfica, 61(3), 623-649.

Diputació de Girona. (2019): XIFRA - Sistema Informació Socieconòmica Local. Retrieved March 25, 2019, from http://www.ddgi.cat/xifra/provincials/activecon/tur_enq_campings.asp?IdMe$\mathrm{nu}=03030804$.

El Pais. (2008): Las reservas del pantano de Boadella suben al 31\%. Retrieved from https://elpais.com/ diario/2008/12/29/catalunya/1230516441_850215.html. 
El País. (2007, July 17): Las cuencas de los ríos, en estado de excepcionalidad por la sequía. Retrieved from https://elpais.com/diario/2007/07/17/catalunya/1184634455_850215.html.

El País. (2008, June 5): La sequía se ceba en el Alt Empordà. Retrieved from https://elpais.com/diario/2008/06/05/catalunya/1212628038_850215.html.

El Periódico. (2008, June 23): La sequía persiste en el Alt Empordà y obliga a llenar piscinas con agua de mar. Retrieved from https://www.elperiodico.com/es/sociedad/20080623/la-sequia-persiste-enel-alt-emporda-y-obliga-a-llenar-piscinas-con-agua-de-mar-39276.

Gabarda-Mallorquí, A., Fraguell, R. M., \& Ribas, A. (2018): Exploring environmental awareness and behavior among guests at hotels that apply water-saving measures. Sustainability (Switzerland), 10(5): https://doi.org/10.3390/su10051305.

Gabarda-Mallorquí, A., Garcia, X., \& Ribas, A. (2017): Mass tourism and water efficiency in the hotel industry: A case study. International Journal of Hospitality Management, 61, 82-93. https://doi. org/10.1016/j.ijhm.2016.11.006.

Gabarda-Mallorquí, A., Ribas, A., \& Daunis-i-Estadella, J. (2015): Desarrollo turístico y gestión eficiente del agua. Una oportunidad para el turismo sostenible en la Costa Brava (Girona): Investigaciones Turísticas, 9, 50-69. https://doi.org/10.14198/INTURI2015.9.03.

Generalitat de Catalunya. (2017): Guia d'establiments i activitats turístiques. Retrieved August 15, 2018, from http://establimentsturistics.gencat.cat/rtcwebguies/AppJava/index.jsp.

Gilg, A., \& Barr, S. (2006): Behavioural attitudes towards water saving? Evidence from a study of environmental actions. Ecological Economics, 57(3), 400-414. https://doi.org/10.1016/j.ecolecon.2005.04.010.

Gómez, B., Armesto, X. A., \& Cors, M. (2017): Percepción del cambio climático y respuestas locales de adaptación: el caso del turismo rural. Cuadernos de Turismo, 39, 287-310. https://doi.org/10.6018/ turismo.39.290571.

Gössling, S., Araña, J. E., \& Aguiar-Quintana, J. T. (2019): Towel reuse in hotels: Importance of normative appeal designs. Tourism Management, 70, 273-283. https://oi.org/10.1016/j.tourman.2018.08.027.

Gössling, S., \& Hall, C. M. (2015): Tourism and water. Channel View Publications.

Gössling, S., \& Peeters, P. (2015): Assessing tourism's global environmental impact 1900 - 2050. Journal of Sustainable Tourism, 23(5), 639-659. https://doi.org/10.1080/09669582.2015.1008500.

Gössling, S., Peeters, P., Hall, C. M., Ceron, J., Dubois, G., Vergne, L., \& Scott, D. (2012): Tourism and water use: Supply, demand, and security. An international review. Tourism Management, 33(1), 1-15. https://doi.org/10.1016/j.tourman.2011.03.015.

Gössling, S., Scott, D., \& Hall, C. M. (2020): Pandemics, tourism and global change: a rapid assessment of COVID-19. Journal of Sustainable Tourism, 1-20. https://doi.org/10.1080/09669582.2020 .1758708 .

Gregory, G. D., \& Leo, M. Di. (2003): Repeated Behavior and Environmental Psychology: The Role of Personal Involvement and Habit Formation in Explaining Water Consumption1. Journal of Applied Social Psychology, 33(6), 1261-1296. https://doi.org/10.1111/j.1559-1816.2003.tb01949.x. 
Hadjikakou, M., Miller, G., Chenoweth, J., Druckman, A., \& Zoumides, C. (2015): A comprehensive framework for comparing water use intensity across different tourist types. Journal of Sustainable Tourism, 23(10), 1445-1467. https://doi.org/10.1080/09669582.2015.1044753.

Han, H., Hsu, L. J. T. J., Lee, J. S., \& Sheu, C. (2011): Are lodging customers ready to go green? An examination of attitudes, demographics, and eco-friendly intentions. International Journal of Hospitality Management, 30(2), 345-355. https://doi.org/10.1016/j.ijhm.2010.07.008.

Han, H., \& Hyun, S. S. (2018a): Eliciting customer green decisions related to water saving at hotels: impact of customer characteristics. Journal of Sustainable Tourism, 26(8), 1437-1452. https://doi.or $\mathrm{g} / 10.1080 / 09669582.2018 .1458857$.

Han, H., \& Hyun, S. S. (2018b): What influences water conservation and towel reuse practices of hotel guests? Tourism Management, 64, 87-97. https://doi.org/10.1016/j.tourman.2017.08.005.

Hu, X., Lovelock, B., Ying, T., \& Mager, S. (2019): Stakeholder Collaboration on Policymaking for Sustainable Water Management in Singapore's Hotel Sector: A Network Analysis. Sustainability, $11(8), 1-19$.

Jarvis, N., \& Ortega, A. P. (2010): The impact of climate change on small hotels in Granada, Spain. Tourism and Hospitality, Planning and Development, 7(3), 283-299. https:/doi.org/10.1080/1479053X.2010.502389.

Kaján, E., \& Saarinen, J. (2013): Tourism, climate change and adaptation: A review. Current Issues in Tourism, 16(2), 167-195. https://doi.org/10.1080/13683500.2013.774323.

Kasim, A. (2009): Managerial attitudes towards environmental management among small and medium hotels in Kuala Lumpur. Journal of Sustainable Tourism, 17(6), 709-725. https://doi. org/10.1080/09669580902928468.

Kelly, J., \& Williams, P. (2007): Tourism destination water management strategies: An eco-efficiency modelling approach. Leisure/Loisir, 31(2), 427-452. https://doi.org/10.1080/14927713.2007.9651 390.

Kim, Y. J., Palakurthi, R., \& Hancer, M. (2012a): The Environmentally Friendly Programs in Hotels and Customers' Intention to Stay: An Online Survey Approach. International Journal of Hospitality and Tourism Administration, 13(3), 195-214. https://doi.org/10.1080/15256480.2012.698169.

Kim, Y. J., Palakurthi, R., \& Hancer, M. (2012b): The Environmentally Friendly Programs in Hotels and Customers' Intention to Stay: An Online Survey Approach. International Journal of Hospitality Eु Tourism Administration, 13(3), 195-214. https://doi.org/10.1080/15256480.2012.698169.

Köberl, J., Prettenthaler, F., \& Bird, D. N. (2016): Modelling climate change impacts on tourism demand: A comparative study from Sardinia (Italy) and Cap Bon (Tunisia): Science of the Total Environment, 543, 1039-1053. https://doi.org/10.1016/j.scitotenv.2015.03.099.

Lam, S. P. (2006): Predicting intention to save water: Theory of planned behavior, response efficacy, vulnerability, and perceived efficiency of alternative solutions. Journal of Applied Social Psychology, 36(11), 2803-2824. https://doi.org/10.1111/j.0021-9029.2006.00129.x. 
Lee, J., Hsu, L. J., Han, H., \& Kim, Y. (2010): Understanding how consumers view green hotels: how a hotel 's green image can influence behavioural intentions, 9582(January 2016): https://doi. org/10.1080/09669581003777747.

Leslie, D. (2001): Serviced accommodation, environmental performance and benchmarks. Journal of Quality Assurance in Hospitality and Tourism, 2(3-4), 127-147. https://doi.org/10.1300/ J162v02n03_08.

Mbasera, M., Du Plessis, E., Saayman, M., \& Kruger, M. (2016): Environmentally-friendly practices in hotels. Acta Commercii, 16(1), 362. https://doi.org/10.4102/ac.v16i1.362.

Millar, M., Mayer, K. J., \& Baloglu, S. (2012): Importance of Green Hotel Attributes to Business and Leisure Travelers. Journal of Hospitality Marketing and Management, 21(4), 395-413. https://doi.org /10.1080/19368623.2012.624294.

Modica, P. D., Altinay, L., Farmaki, A., Gursoy, D., \& Zenga, M. (2020): Consumer perceptions towards sustainable supply chain practices in the hospitality industry. Current Issues in Tourism, 23(3), 358-375. https://doi.org/10.1080/13683500.2018.1526258.

Moreo, A. P. (2008): Green consumption in the hotel industry: an examination of consumer attitudes.

Morrison, C., \& Pickering, C. M. (2013): Perceptions of climate change impacts, adaptation and limits to adaption in the Australian Alps: the ski-tourism industry and key stakeholders. Journal of Sustainable Tourism, 21(2), 173-191. https://doi.org/10.1080/09669582.2012.681789.

Nikolaou, I. E., Vitouladitis, H., \& Tsagarakis, K. P. (2012, June): The willingness of hoteliers to adopt proactive management practices to face energy issues. Renewable and Sustainable Energy Reviews. https://doi.org/10.1016/j.rser.2012.01.061.

Oficina Catalana del Canvi Climàtic. (2013): Estratègia catalana d'adaptació al canvi climàtic. Barcelona. Retrieved from http://medacc-life.eu/sites/medacc-life.eu/files/docuemnts/escacc.pdf.

Olcina Cantos, J. (2012): Turismo y cambio climático: una actividad vulnerable que debe adaptarse. Investigaciones Turísticas, 4, 1-34. Retrieved from https://rua.ua.es/dspace/bitstream/10045/25736/1/ Investigaciones_Turisticas_4_01.pdf.

Page, S. J., Essex, S., \& Causevic, S. (2014): Tourist attitudes towards water use in the developing world: A comparative analysis. Tourism Management Perspectives, 10, 57-67. https://doi.org/10.1016/j.tmp.2014.01.004.

Pascual, D., Zabalza Martínez, J., Funes, I., Vicente-Serrano, S. M., Pla, E., Aranda, X. Biel, C. (2016): Impacts of climate and global change on the environmental, hydrological and agricultura systems in the LIFE MEDACC case study basins. Delverable 14. Retrieved from www.medacc-life.euwww.medacc-life.eu.

Pla, E., Pascual, D., Borràs, G., Cantos, G., Funes, I., Aranda, X., Biel, C. (2017): Three action plans for climate change adaptation in the LIFE MEDACC case study basins. Deliverable 15. Retrieved from www.medacc-life.euwww.medacc-life.eu.

Ribas, A., \& Saurí, D. (2010): 2008, l'any de la sequera. Anuari Territorial de Catalunya, 2008, 13-16.

Roberts, J. A. (1996): Green consumers in the 1990s: Profile and implications for advertising. Journal of Business Research, 36(3), 217-231. https://doi.org/10.1016/0148-2963(95)00150-6. 
Tekken, V., \& Kropp, J. P. (2015): Sustainable water management - perspectives for tourism development in north-eastern Morocco. Tourism Management Perspectives, 16, 325-334. https://doi. org/10.1016/j.tmp.2015.09.001.

UNEP. (2019): Retrieved August 21, 2019, from https://www.unenvironment.org/es/explore-topics/ agua.

Ventura, M., Ribas, A., \& Saurí, D. (2000): Gestión del agua y conflictividad social en la cuenca del río Muga (Alt Empordà): Geographicalia, 38, 59-75.

Vicente-Serrano, S., Pascual, D., Pla, E., Zabalza, J., Borràs, G., Cantos, G., Funes, I. (2016): Historical trends in climate, land use and water demands. Deliverable 12. Retrieved from www.medacc-life.eu.

Wang, W.-C., Lin, C.-H., Lu, W.-B., \& Lee, S.-H. (2019): When destination attractiveness shifts in response to climate change: tourists' adaptation intention in Taiwan's Kenting National Park. Current Issues in Tourism, 22(5), 522-543. https://doi.org/10.1080/13683500.2018.1437715.

Warren, C., Becken, S., \& Coghlan, A. (2017): Using persuasive communication to co-create behavioural change-engaging with guests to save resources at tourist accommodation facilities. Journal of Sustainable Tourism, 25(7), 935-954. https://doi.org/10.1080/09669582.2016.1247849.

Cómo citar este artículo:

Torres-Bagur, M., Ribas, A., Vila-Subirós, J. (2020). Usos del agua y prácticas de ahorro hídrico de los turistas en la cuenca del río Muga (Girona). Cuadernos de Geografía, 104, 131-152.

https://doi.org/10.7203/cguv.104.16785

\section{(c) $(1) \Theta \Theta$}

Este obra está bajo una licencia de Creative Commons Reconocimiento-NoComercial-SinObraDerivada 4.0 Internacional. 
